\title{
A Case of Acute Spontaneous Tumor Lysis Syndrome and NeW Diagnosis OF BURKITT'S LYMPHOMA
}

Ryan D. Gentzler, MD

\section{Introduction}

Tumor lysis syndrome is a well-described phenomenon characterized by elevated serum levels of calcium, uric acid, potassium, phosphate, and lactate dehydrogenase due to lysis of tumor cells and release of intracellular contents. Acute kidney injury may occur as the result of precipitation of intrarenal calcium phosphate salts related to the rapid destruction of a large number of tumor cells. ${ }^{1}$ Tumor lysis syndrome most often occurs during induction chemotherapy for aggressive leukemia or lymphoma, particularly those with large tumor burden.1 While tumor lysis syndrome is more commonly seen in patients receiving chemotherapy, it can occur spontaneously and has been described in aggressive malignancies, such as AML, ${ }^{2}$ Burkitt's lymphoma in children 1 and adults, ${ }^{3}$ and in solid malignancies, such as breast cancer. ${ }^{4}$ This case describes a patient who presented with a neck mass and spontaneous tumor lysis syndrome.

\section{Case Presentation}

A 76-year-old male with a past medical history of hypertension, hyperlipidemia, and coronary artery disease presented with epistaxis and a neck mass that was first noted four weeks prior to presentation. In addition, the patient noticed gingival bleeding when brushing his teeth, malaise, decreased appetite, and night sweats, all of which developed during the previous week. His exam was significant for an ulcer with scab on his left buccal mucosa, a 6-cm left neck mass that was firm and non-tender, and several areas of ecchymosis on his arms bilaterally. There was no axillary or inguinal lymphadenopathy. The remainder of the exam was unremarkable.

A neck ultrasound prior to admission showed multiple left cervical cystic and solid masses consistent with necrotic lymph nodes. The patient also had an enlarged right cervical lymph node. He was admitted with a diagnosis of left neck mass suspicious for malignancy and acute kidney injury.

On admission, laboratory data showed white blood cell count of $5,300 / \mu \mathrm{L}$, a platelet count of $3,000 / \mu \mathrm{L}$, hemoglobin $12.5 \mathrm{~g} /$ $\mathrm{dL}$, and a mean corpuscular volume of $94 \mathrm{fL}$. The white cell count differential included $23 \%$ neutrophils, $10 \%$ bands, $28 \%$ lymphocytes, $13 \%$ atypical lymphocytes, $2 \%$ monocytes, $1 \%$ metamyelocytes, $5 \%$ myelocytes, $2 \%$ promyelocytes, $16 \%$ blasts, and 3.4/100 WBC nucleated red cells. Other significant laboratory data included potassium of $4.5 \mathrm{mmol} / \mathrm{L}$, blood urea nitrogen (BUN) of $46 \mathrm{mg} / \mathrm{dL}$, creatinine of $1.6 \mathrm{mg} / \mathrm{dL}$, total bilirubin of $2.1 \mathrm{mg} / \mathrm{dL}$, direct bilirubin of $0.7 \mathrm{mg} / \mathrm{dL}$, uric acid of $17.4 \mathrm{mg} / \mathrm{dL}$, and lactate dehydrogenase (LDH) of $4795 \mathrm{IU} / \mathrm{L}$.

The patient's elevated LDH, uric acid, and creatinine raised suspicion of tumor lysis syndrome, so IV fluid hydration and allopurinol were initiated. A bone marrow biopsy was performed for diagnosis of suspected lymphoma. The patient's creatinine, $\mathrm{LDH}$, and uric acid levels continued to rise, and treatment with rasburicase was initiated given the concern for renal failure. In less than 12 hours, the patient's creatinine increased from $2.6 \mathrm{mg} / \mathrm{dL}$ to $3.5 \mathrm{mg} / \mathrm{dL}$ and potassium increased from 5.2 $\mathrm{mmol} / \mathrm{L}$ to $6.1 \mathrm{mmol} / \mathrm{L}$. The patient suffered cardiac arrest before dialysis could be initiated. Although he was initially resuscitated, care was withdrawn the same day per the patient's and family's wishes.

The final bone marrow biopsy results, including flow cytometry and FISH analysis, revealed solid monotonous sheets of large atypical lympohoid cells with clear cytoplasmic vacuoles, which accounted for the vast majority of nucleated bone marrow cells. The cells demonstrated a monoclonal B-cell proliferation with translocations at 8;22 involving the $\mathrm{C}$-myc gene, consistent with the diagnosis of Burkitt's lymphoma. These results were obtained several days after the patient had expired.

\section{Discussion}

Tumor lysis syndrome is a familiar entity to physicians caring for patients receiving chemotherapy for treatment of leukemias and lymphomas. This syndrome is most commonly encountered upon initiation of chemotherapy in a patient for the first time, but it can occur in any patient with extensive tumor burden, including patients with solid malignancies. Patients may exhibit hypercalcemia, hyperkalemia, hyperphosphatemia, hyperuricemia and acute renal failure. Often prophylactic intravenous fluid hydration and administration of allopurinol or rasburicase are initiated prior to giving chemotherapy to reduce the risk of developing renal failure and fatal arrhythmias secondary to hyperkalemia. In addition, frequent monitoring of serum potassium levels allows for treatment of hyperkalemia, should it develop.

In the case presented above, tumor lysis syndrome was not an anticipated event since chemotherapy was not being given at the time of its development. Moreover, the patient had not been diagnosed with a malignancy at the time of development of the syndrome. Although it has been described before in the literature, this uncommon presentation of spontaneous tumor lysis syndrome could be easily missed. Knowing that spontaneous tumor lysis syndrome can occur, it seems reasonable to obtain serum lactate dehydrogenase and serum uric acid levels in patients suspected of having a new diagnosis of a hematologic malignancy even before the formal diagnosis is made. It is important to initiate standard treatment of IV fluid hydration and allopurinol or rasburicase as early as possible when tumor lysis syndrome is suspected. 


\section{References}

1. Cohen LF, Balow JE, Magrath IT, et al. Acute Tumor Lysis Syndrome. American Journal of Medicine. 1980; 68:486-491.

2. Lotfi M, Brandwein JM. Spontaneous Acute Tumor Lysis Syndrome in Acute Myeloid Leukemia? A Single Case Report with Discussion of the Literature. Leukemia and Lymphoma. 1998; 29:625-628.
3. Jasek AM, Day HJ. Acute Spontanous Tumor Lysis Syndrome. American Journal of Hematology. 1994; 47:129-131.

4. Sklarin NT, Markham M. Spontaneous Recurrent Tumor Lysis Syndrome in Breast Cancer. American Jrounal of Clinical Oncology. 1995; 18(1): 71-73.

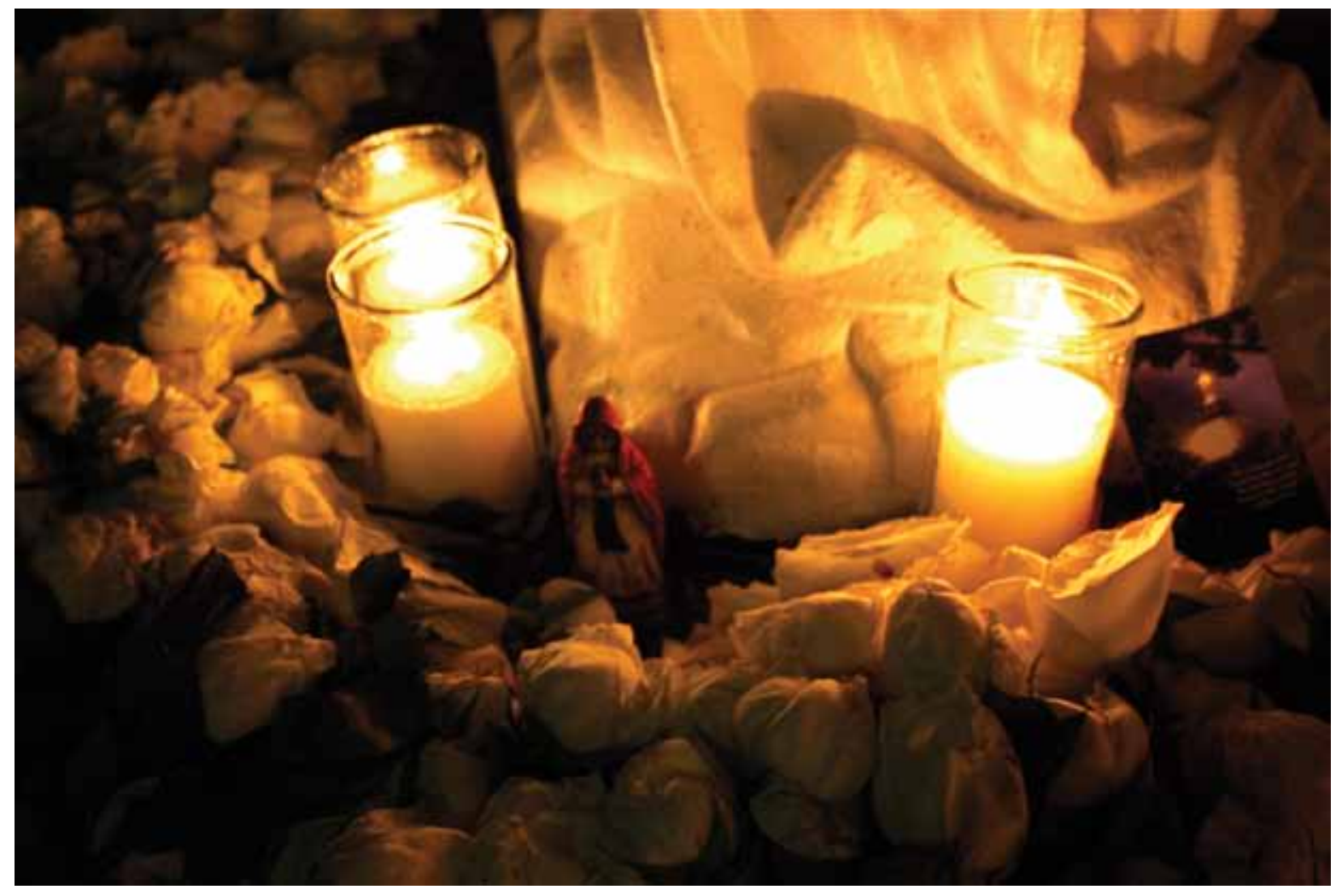

"Notre Dame"

photograph by Cecilia Kelly, MD 